Portland State University

PDXScholar

$11-1-2019$

\title{
On the Equality Case of the Ramanujan Conjecture for Hilbert Modular Forms
}

Liubomir Chiriac

Portland State University, chiriac@pdx.edu

Follow this and additional works at: https://pdxscholar.library.pdx.edu/mth_fac

Part of the Number Theory Commons

Let us know how access to this document benefits you.

Citation Details

Chiriac, L. (2019). On the equality case of the Ramanujan Conjecture for Hilbert modular forms.

International Journal of Number Theory, 1-8.

This Post-Print is brought to you for free and open access. It has been accepted for inclusion in Mathematics and Statistics Faculty Publications and Presentations by an authorized administrator of PDXScholar. Please contact us if we can make this document more accessible: pdxscholar@pdx.edu. 


\title{
ON THE EQUALITY CASE OF THE RAMANUJAN CONJECTURE FOR HiLBERT MODUlaR FORMS
}

\author{
LiUbOMiR CHIRIAC
}

\begin{abstract}
The generalized Ramanujan Conjecture for cuspidal unitary automorphic representations $\pi$ on $\mathrm{GL}(2)$ asserts that $\left|a_{v}(\pi)\right| \leq 2$. We prove that this inequality is strict if $\pi$ is generated by a CM Hilbert modular form of parallel weight two and $v$ is a finite place of degree one. Equivalently, the Satake parameters of $\pi_{v}$ are necessarily distinct. We also give examples where the equality case does occur for primes of degree two.
\end{abstract}

\section{Introduction}

Let $\pi=\otimes_{v} \pi_{v}$ be a cuspidal unitary automorphic representation of $\mathrm{GL}_{2}\left(\mathbb{A}_{F}\right)$, where $\mathbb{A}_{F}$ is the ring of adèles of a number field $F$. For almost all finite places $v$ the representation $\pi_{v}$ of the local group $\mathrm{GL}_{2}\left(F_{v}\right)$ is unramified. For such $v$, the associated semisimple conjugacy class in $\mathrm{GL}_{2}(\mathbb{C})$ is given by a diagonal matrix

$$
\left(\begin{array}{cc}
\alpha_{v} & 0 \\
0 & \beta_{v}
\end{array}\right),
$$

where $\alpha_{v}$ and $\beta_{v}$ are complex numbers called the Satake parameters of $\pi_{v}$. The trace of this class is denoted by $a_{v}(\pi)$.

The generalized Ramanujan Conjecture (GRC) predicts that all unramified $\pi_{v}$ are tempered, i.e.,

$$
\left|\alpha_{v}\right|=\left|\beta_{v}\right|=1
$$

The GRC is known to be true in several special cases, such as when $\pi$ is attached to a classical holomorphic modular form of integral weight or to a holomorphic Hilbert modular form having integer weights $\geq 2$ and all of the same parity (see Livné [15] for a detailed account on the latter). In general, one knows that $\left|\alpha_{v}\right|,\left|\beta_{v}\right| \leq(N v)^{7 / 64}$ by the work of Kim and Sarnak [13] (over $\mathbb{Q}$ ), and Blomer and Brumley [3] (over arbitrary number fields). In another direction, Ramakrishnan [17] proved that at least 9/10 of the local components satisfy the GRC. This bound was further improved by Kim and Shahidi [14] to $34 / 35 \approx 0.971$. 
An equivalent way $\left.\right|^{1}$ of stating the GRC is that $\left|a_{v}(\pi)\right| \leq 2$. In the context of classical or Hilbert modular forms this is also referred to as the Ramanujan-Petersson conjecture. The primary goal of the present paper is to address the sharpness of this inequality. In other words, we are interested whether the Satake parameters of $\pi$ at some $v$ can be equal to each other. Our main result is the following:

Theorem 1. Let $F$ be a totally real number field. Let $\pi=\otimes_{v} \pi_{v}$ be a cuspidal automorphic representation of $\mathrm{GL}_{2}\left(\mathbb{A}_{F}\right)$ associated to a holomorphic CM Hilbert modular newform $\mathbf{f}$ of parallel weight two and trivial character. Let $v$ be a place of $F$ of degree one that does not divide the level of $\mathbf{f}$ and the different ideal of $\mathrm{F}^{2}$ Then the Satake parameters of $\pi_{v}$ are necessarily distinct.

If $\pi$ is attached to a classical modular form of weight $k \geq 2$, Coleman and Edixhoven [4] have shown that the Satake parameters are always distinct, provided that the action of the crystalline Frobenius is semisimple. This is known for $k=2$ unconditionally, whereas for higher weights it would follow from the Tate conjectures [19]. On the other hand, using some congruences of Hatada [9], it is not too difficult to see (cf. Gouvêa [8] that the conclusion of Theorem 1 holds for classical modular forms of level one.

To prove Theorem 1 we distinguish two cases. If the degree $[F: \mathbb{Q}]$ is odd or if $[F: \mathbb{Q}]$ is even and $\pi$ has some square-integrable local component one can transfer to a quaternionic Shimura curve via the Jacquet-Langlands correspondence and then proceed as in [4] - this is the content of Section 2. Thus, the novelty here is when $[F: \mathbb{Q}]$ is even and there are no square-integrable local components. In this case, we make use of a construction based on the Grunwald-Wang theorem for which we have to assume that $\mathbf{f}$ has complex multiplication (CM), i.e., it arises from a Hecke character of a totally imaginary quadratic extension of $F$. This way, we reduce the problem to the previous case (cf. Section 3).

In general, for a regular algebraic cuspidal automorphic representation $\pi$, the set of places $v$ where $\left|a_{v}(\pi)\right|=2$ has density zero. Indeed, this follows directly from the SatoTate conjecture, which has been known from the classical work of Deuring [6] and Hecke [10] in the CM case, and has been proved more recently in the non-CM case [2]. At the same time, we do emphasize that the equality case of GRC can occur at places of degree greater than one. Section 4 contains a general recipe of producing Hilbert newforms that have extremal Hecke eigenvalues at primes $v$ of degree two. This is achieved by base change lifting on GL(2) under a real quadratic extension $F / \mathbb{Q}$, and specializing at certain inert primes. To illustrate the existence of extremal Hecke eigenvalues, we give sample numerical computations for the modular elliptic curve $X_{0}(14)$ and its base change lift to $\mathbb{Q}(\sqrt{17})$.

\footnotetext{
${ }^{1}$ If $n \geq 3$, the temperedness condition for $\pi$ on $\mathrm{GL}_{n}\left(\mathbb{A}_{F}\right)$ clearly implies, but is not equivalent to, $\left|a_{v}(\pi)\right| \leq n$.

${ }^{2}$ The fact $v$ does not divide the level of $\mathbf{f}$ or the different ideal of $F$ ensures that $\pi_{v}$ is a spherical representation.
} 


\section{The Jacquet-Langlands condition}

In this section we do not assume that $\pi$ is of CM type, but instead suppose that it has a square-integrable local component when $[F: \mathbb{Q}]$ is even. This ensures the existence of an abelian variety that arises as a quotient of the Jacobian of a suitably defined Shimura curve, via the Jacquet-Langlands correspondence. As a result, one can exploit the semisimplicity of the Frobenius endomorphism in this case.

Proposition 2. Let $F$ be a totally real number field. Let $\pi=\otimes_{v} \pi_{v}$ be a cuspidal automorphic representation of $\mathrm{GL}_{2}\left(\mathbb{A}_{F}\right)$ associated to a Hilbert modular newform $\mathbf{f}$ of parallel weight two. If $[F: \mathbb{Q}]$ is even, assume that there exists a finite place of $F$ where the local component of $\pi$ is square-integrable. Let $v$ be a place of $F$ of degree one that does not divide the level of $\mathbf{f}$ and the different ideal of $F$. Then the Satake parameters of $\pi_{v}$ are necessarily distinct.

Proof. By global class field theory, the set of places at which a quaternion algebra $B$ ramifies is finite and of even cardinality. Conversely, given an even number of places there exists a unique quaternion algebra that ramifies at precisely those places. Now, let $B$ be the quaternion algebra over $F$ with the following properties:

- $B$ splits at only one infinite place;

- if $[F: \mathbb{Q}]$ is odd, $B$ is unramified at every finite place;

- if $[F: \mathbb{Q}]$ is even, the finite place where $\pi$ is square-integrable (by assumption) is the unique finite place where $B$ is ramified.

Denote by $\operatorname{ram}(B)$ the set of places at which $B$ is ramified, and by $\operatorname{disc}(B)$ the product of all the finite places in $\operatorname{ram}(B)$ (which, by definition, is the discriminant of $B)$. Let $\mathfrak{n}^{\prime}$ be an ideal such that $\mathfrak{n}=\mathfrak{n}^{\prime} \operatorname{disc}(B)$, where $\mathfrak{n}$ is the level of $\mathbf{f}$. By the Jacquet-Langlands correspondence, there exists a cuspidal automorphic representation $\pi^{\prime}$ of $B\left(\mathbb{A}_{F}\right)^{\times}$such that $\pi_{v} \simeq \pi_{v}^{\prime}$ for every $v \notin \operatorname{ram}(B)$.

To get the prescribed level structure, we take an Eichler order $\mathcal{O}\left(\mathfrak{n}^{\prime}\right)$ in $B$ of level $\mathfrak{n}^{\prime}$ and consider the group $\mathcal{O}^{1}\left(\mathfrak{n}^{\prime}\right)=\left\{\theta \in \mathcal{O}\left(\mathfrak{n}^{\prime}\right) \mid \operatorname{nrd}(\theta)=1\right\}$, i.e., the elements of reduced norm 1 . Since our $B$ is split at exactly one real place, there is a map

$$
B \hookrightarrow B \otimes_{\mathbb{Q}} \mathbb{R} \simeq M_{2}(\mathbb{R}) \times \mathbb{H}^{n-1}
$$

where $\mathbb{H}$, as usual, are the Hamilton quaternions. Letting $\iota: B \hookrightarrow M_{2}(\mathbb{R})$ be the projection onto the first factor, we obtain an arithmetic Fuchsian group (i.e., a discrete subgroup of $\mathrm{PSL}_{2}(\mathbb{R})$ )

$$
\Gamma^{B}\left(\mathfrak{n}^{\prime}\right)=\iota\left(\mathcal{O}^{1}\left(\mathfrak{n}^{\prime}\right)\right) \subset \mathrm{GL}_{2}^{+}(\mathbb{R})
$$

This group acts by linear fractional transformations on the upper half-plane $\mathfrak{H}$ and the compact quotient $\Gamma^{B}\left(\mathfrak{n}^{\prime}\right) \backslash \mathfrak{H}$ can be viewed as the complex points of the connected component of a Shimura curve $C_{B}$, which has a canonical model defined over $F$. 
At this point, one can argue exactly as in [4 to get the conclusion. For the convenience of the reader, we sketch the argument below.

Let $K_{\mathbf{f}}$ be the number field generated by all the Fourier coefficients of $\mathbf{f}$, and let $\mathcal{O}_{K_{\mathbf{f}}}$ be its ring of integers. As shown by Hida ([11] Theorem 4.4), there exists an abelian variety $A_{\mathbf{f}}$ defined over $F$, which arises as a quotient of the Jacobian of $C_{B}$. To get an action of all of $\mathcal{O}_{K_{\mathbf{f}}}$ take $A=A_{\mathbf{f}} \otimes \mathcal{O}_{K_{\mathbf{f}}}$. If $v$ is a prime of $F$ of degree one whose residual characteristic $p$ is prime to the level of $\mathbf{f}$ and $\operatorname{disc}(F)$, the abelian variety $A$ has good reduction at $v$. Denote by $A_{v}$ the reduction of $A$ modulo $v$. The endomorphism ring $\operatorname{End}\left(A_{v}\right)$ of $A_{v}$ is semisimple, so the Frobenius endomorphism Frob ${ }_{v}$ acts on the $\ell$-adic Tate module $(\ell \neq p)$ as a semisimple linear operator.

Assume by contradiction that the Satake parameters of $\pi_{v}$ are equal, i.e.,

$$
\alpha_{v}=\beta_{v}=\lambda .
$$

Then $a_{v}(\pi)=\alpha_{v}+\beta_{v}=2 \lambda$, and the residual degree $q_{v}=\alpha_{v} \cdot \beta_{v}=\lambda^{2}$ is equal to $p$, since $v$ has degree one. The Eichler-Shimura congruence relation implies that the characteristic polynomial of $\operatorname{Frob}_{v}$ is $T^{2}-a_{v}(\pi) T+q_{v}$, and thus $\left(\operatorname{Frob}_{v}-\lambda\right)^{2}=0$ in the endomorphism ring. By the semisimplicity of $\operatorname{Frob}_{v}$, we infer that it must act as the multiplication by the scalar $\lambda$. In other words,

$$
\operatorname{Frob}_{v}=\lambda \text { in } \operatorname{End}\left(A_{v}\right) .
$$

Consider the first de Rham cohomology group $M$ of the associated Néron model $\mathcal{A}$ of $A$ over $\mathcal{O}_{v}$. It admits a Hodge filtration $\operatorname{Fil}^{1} M:=H^{0}\left(\mathcal{A}, \Omega^{1}\right) \subset M$. Since $\operatorname{Fil}^{1}(M)$ is locally isomorphic to $\mathcal{O}_{K_{f}} \otimes \mathcal{O}_{v}$ and $\lambda^{2}=p$ we get that $p$ does not divide $\lambda$, so $\mathrm{Fil}^{1}(M) \otimes \mathbb{F}_{v}$ is not annihilated by $\lambda$. However, Frob $v$ has differential zero, so by the previous paragraph we get that $\lambda$ does annihilate $H^{0}\left(A_{v}, \Omega^{1}\right)=\mathrm{Fil}^{1}(M) \otimes \mathbb{F}_{v}$, which is a contradiction.

\section{Proof of the main result}

Now we assume that the Hilbert modular form $\mathbf{f}$ has complex multiplication by an imaginary quadratic extension $K / F$ with the associated Hecke character $\chi$. Under this assumption we will settle the case not covered by Proposition 2 namely, when $[F: \mathbb{Q}]$ is even and the automorphic representation $\pi$ generated by $\mathbf{f}$ has no discrete series component at a finite place; so one cannot move to a Shimura curve directly. Our strategy is to construct a certain character $\mu$ over $K$ defined by some local relations such that the automorphic representation $\pi^{\prime}$ of $\mathrm{GL}_{2}\left(\mathbb{A}_{F}\right)$ corresponding to $\chi \mu$ will have the following two properties:

$\left(P_{1}\right) \pi_{v}$ and $\pi_{v}^{\prime}$ have the same pair of Satake parameters.

$\left(P_{2}\right) \pi^{\prime}$ has a square-integrable local component at some auxiliary finite place $v^{\prime}$ of $F$. 
This way, by switching from $\pi$ to $\pi^{\prime}$ we reduce the problem to the situation treated in the previous section.

Proof of Theorem 1. Note that $v$ cannot be inert in $K$, for otherwise $a_{v}(\pi)=0$. Therefore, we can assume that $v$ splits in $K$. Denote by $\mathfrak{q}$ and $\mathfrak{q}^{\prime}$ the primes above $v$ in $K$. If $\pi$ has a discrete series component $\pi_{u}$ then the conclusion follows from Proposition 2 , So assume otherwise, and pick an auxiliary finite place $v^{\prime}$ of $F$ that remains inert in $K$ (for simplicity, we keep the same notation for the prime in $K$ above $v^{\prime}$ ). Consider the set $S=\left\{\mathfrak{q}, \mathfrak{q}^{\prime}, v^{\prime}\right\}$ of places of $K$. To each element of $S$ we shall associate a local character as follows:

- For $\mathfrak{q}$ and $\mathfrak{q}^{\prime}$, simply take the trivial characters of $K_{\mathfrak{q}}^{\times}$and $K_{\mathfrak{q}^{\prime}}^{\times}$.

- For $v^{\prime}$, choose $\lambda$ to be a quadratic character of $K_{v^{\prime}}^{\times}$such that $\lambda \neq \lambda \circ c$, where $c$ is the non-trivial element of $\operatorname{Gal}\left(K_{v^{\prime}} / F_{v^{\prime}}\right)$. More concretely, we will take $\lambda$ to be the quadratic character of $K_{v^{\prime}}^{\times}$attached to its unique unramified quadratic extension.

By the Grunwald-Wang theorem (Theorem 5 in Chapter X of [1]) there exists a finite order global character $\mu$ of $K$ such that

$$
\mu_{\mathfrak{q}}=\mu_{\mathfrak{q}^{\prime}}=1, \mu_{v^{\prime}}=\lambda \text { and } \mu_{\infty}=1 .
$$

Let $\pi^{\prime}=A I_{K}^{F}(\chi \mu)$ be the image of $\chi \mu$ under the automorphic induction map from the set of automorphic representation of $\mathrm{GL}_{1}\left(\mathbb{A}_{K}\right)$ to the set of automorphic representation of $\mathrm{GL}_{2}\left(\mathbb{A}_{F}\right)$. Note that $\pi^{\prime}$ is cuspidal (cf. Example 3 in [18]).

Lemma 3. $\pi^{\prime}$ satisfies the properties $\left(P_{1}\right)$ and $\left(P_{2}\right)$.

Proof. Since $v=\mathfrak{q} \mathfrak{q}^{\prime}$, the coefficient $a_{v}(\pi)$ can be written as

$$
a_{v}(\pi)=\chi_{\mathfrak{q}}(\omega)+\chi_{\mathfrak{q}^{\prime}}(\omega)
$$

where $\omega$ is an uniformizer of $F_{v} \cong K_{\mathfrak{q}} \cong K_{\mathfrak{q}^{\prime}}$. The condition $\mu_{\mathfrak{q}}=\mu_{\mathfrak{q}^{\prime}}=1$ implies that $\chi_{\mathfrak{q}} \mu_{\mathfrak{q}}=\chi_{\mathfrak{q}}$ and $\chi_{\mathfrak{q}^{\prime}} \mu_{\mathfrak{q}^{\prime}}=\chi_{\mathfrak{q}^{\prime}}$. Therefore $a_{v}(\pi)=a_{v}\left(\pi^{\prime}\right)$.

Consider the quadratic character $\varepsilon$ of $F$ corresponding to $K / F$ such that $f=f \otimes \varepsilon$. By hypothesis, $\pi$ has trivial central character, i.e., $\left.\chi\right|_{F}=\varepsilon$. To establish property $\left(P_{1}\right)$ it suffices to show that $\pi^{\prime}$ has trivial character at $v$. This is equivalent to showing that $\chi_{v} \mu_{v}=\varepsilon_{v}$, which is true because $v$ splits.

Moreover, by our construction it follows that

$$
\pi_{v^{\prime}}^{\prime}=\operatorname{Ind}_{K_{v^{\prime}}}^{F_{v^{\prime}}}\left(\chi_{v^{\prime}} \mu_{v^{\prime}}\right)=\operatorname{Ind}_{K_{v^{\prime}}}^{F_{v^{\prime}}}\left(\chi_{v^{\prime}} \lambda\right)
$$

Since we assumed that no local component of $\pi$ is square-integrable, $\pi_{v}$ is not supercuspidal, so Theorem 4.6 of [12] implies that $\chi_{v^{\prime}}=\chi_{v^{\prime}} \circ c$. At the same time, the character $\lambda$ has the defining property that $\lambda \neq \lambda \circ c$, hence $\chi_{v^{\prime}} \lambda \neq \chi_{v^{\prime}} \lambda \circ c$. By the same theorem of [12], the local component $\pi_{v^{\prime}}^{\prime}$ is supercuspidal, and thus property $\left(P_{2}\right)$ also holds. 
Using Lemma 3 we can replace $\pi$ with a related $\pi^{\prime}$, without changing the Satake parameters at $v$, such that $\pi^{\prime}$ satisfies the conditions of Proposition 2. In conclusion, these Satake parameters of $\pi_{v}$ (and $\pi_{v}^{\prime}$ ) are distinct.

\section{Examples of extremal Hecke eigenvalues}

In this section we give a general recipe of constructing cuspidal automorphic representations on GL(2) where the equality case of the GRC occurs for certain places $v$ of degree two. The underlying idea is that inert supersingular primes can be "lifted" to produce extremal Hecke eigenvalues, i.e., eigenvalues of largest possible absolute value permitted by the Weil bounds. The example below was kindly explained to us by Emerton and is originally due to Kottwitz.

Example 4. Start with an elliptic curve $E$ over $\mathbb{Q}$ and a prime $p$ of good supersingular reduction, i.e., the trace of Frobenius $a_{p}(E)$ is 0 . By a classical result of Elkies [7], it is known that there exist infinitely many such $p$. Pick any real quadratic field $F$ in which $p$ is inert. Again, for a given $p$, there are infinitely many quadratic fields with this property.

Let $f$ be a newform associated to $E$ by the modularity theorem, so that the $p$-th Fourier coefficient $a_{p}(f)$ of $f$ is $a_{p}(E)$. Under the quadratic base change from $\mathbb{Q}$ to $F$, one can lift $f$ to a parallel weight two Hilbert modular form $\mathbf{f}$ for $F$. Denote by $\mathfrak{p}$ the prime of $F$ lying above $p$. Since $p$ is inert in $F$, it follows that the ideal $\mathfrak{p}$ has norm $N(\mathfrak{p})=p^{2}$ and

$$
\left|a_{\mathfrak{p}}(\mathbf{f})\right|=\left|a_{p}(f)^{2}-2 p\right|=2 p .
$$

Therefore, if $\pi$ is the cuspidal automorphic representation generated by $\mathbf{f}$ then

$$
\left|a_{\mathfrak{p}}(\pi)\right|=a_{\mathfrak{p}}(\mathbf{f}) / N(\mathfrak{p})^{1 / 2}=2,
$$

so the Satake parameters of $\pi$ at $\mathfrak{p}$ are equal.

For an explicit numerical example, we consider the elliptic curve $E$ given by the minimal Weierstrass equation

$$
y^{2}+x y+y=x^{3}+4 x-6,
$$

which ${ }^{3}$ is a model for the modular curve $X_{0}(14)$. Associated to the isogeny class of $E$ is the unique newform $f$ of weight 2 and level 14 .

\footnotetext{
${ }^{3}$ The elliptic curve $E$ has Cremona label 14a1 (cf. [5]).
} 
We can write $f$ as a product of eta functions

$$
\begin{aligned}
f(z) & =\eta(z) \eta(2 z) \eta(7 z) \eta(14 z) \\
& =q \prod_{n=1}^{\infty}\left(1-q^{n}\right)\left(1-q^{2 n}\right)\left(1-q^{7 n}\right)\left(1-q^{14 n}\right) \\
& =q-q^{2}-2 q^{3}+q^{4}+2 q^{6}+q^{7}-q^{8}+q^{9}-2 q^{12}-4 q^{13}-q^{14}+\ldots
\end{aligned}
$$

with $q=e^{2 \pi i z}$. Denote by $\mathbf{f}$ the base change lift of $f$ from $\mathbb{Q}$ to $F=\mathbb{Q}(\sqrt{17})$.

Using Pari/GP [16] we find that the first few supersingular primes of $E$ which are inert in $F$ are given by the following table:

\begin{tabular}{c|ccccccc}
$p$ & 5 & 11 & 23 & 71 & 503 & 1031 & 1637 \\
\hline$a_{p}(f)$ & 0 & 0 & 0 & 0 & 0 & 0 & 0 \\
\hline$N(\mathfrak{p})$ & 25 & 121 & 529 & 5041 & 253009 & 1062961 & 2679769 \\
\hline$a_{\mathfrak{p}}(\mathbf{f})$ & -10 & -22 & -46 & -142 & -1006 & -2062 & -3274
\end{tabular}

In each case $\left|a_{\mathfrak{p}}(\mathbf{f})\right|=2 N(\mathfrak{p})^{1 / 2}$, so these Hecke eigenvalues of $\mathbf{f}$ are extremal.

The above construction can be summarized in a slightly more general setting:

Lemma 4. Suppose $K / F$ be a quadratic extension of number fields. Let $\pi$ be a unitary cuspidal automorphic representation of $\mathrm{GL}_{2}\left(\mathbb{A}_{F}\right)$, and let $\pi^{\prime}$ be its base change lift to $\mathrm{GL}_{2}\left(\mathbb{A}_{K}\right)$. Let $v$ be a place of $F$ such that $\pi_{v}$ is unramified. Assume that $v$ is inert in $K$, and denote by $w$ the prime of $K$ lying over $v$. Then $\left|a_{w}\left(\pi^{\prime}\right)\right|=2$ if and only if either $\left|a_{v}(\pi)\right|=2$ or $a_{v}(\pi)=0$.

Proof. Assume that $\left\{\alpha_{v}, \beta_{v}\right\}$ are the Satake parameters of $\pi_{v}$, i.e., the unramified representation $\pi_{v}$ corresponds to the semisimple conjugacy class with diagonal entries $\left\{\alpha_{v}, \beta_{v}\right\}$. Since $v$ is inert in $K$, the Satake parameters of the base change $\pi^{\prime}$ at the prime $w$ over $v$ are $\left\{\alpha_{v}^{2}, \beta_{v}^{2}\right\}$ (cf. Example 6 in [18]). Therefore,

$$
a_{w}\left(\pi^{\prime}\right)=\alpha_{v}^{2}+\beta_{v}^{2}=\left(\alpha_{v}+\beta_{v}\right)^{2}-2 \alpha_{v} \beta_{v}=a_{v}(\pi)^{2}-2
$$

and the conclusion follows.

\section{Acknowledgments}

I would like to thank Dinakar Ramakrishnan for suggesting this problem and for sharing many useful ideas on the subject. I am grateful to Matt Emerton, from whom I learned the construction in Example 4. Thanks are also due to Andrei Jorza and the referee for helpful comments which improved the exposition. 


\section{References}

[1] E. Artin, J. Tate, Class field theory. Reprinted with corrections from the 1967 original. AMS Chelsea Publishing, Providence, RI, 2009.

[2] T. Barnet-Lamb, T. Gee, D. Geraghty The Sato-Tate conjecture for Hilbert modular forms, J. Amer. Math. Soc. 24 (2011), no. 2, 411-469.

[3] V. Blomer, F. Brumley, On the Ramanujan conjecture over number fields, Ann. of Math. (2) 174 (2011), no. 1, 581-605.

[4] R.F. Coleman, B. Edixhoven, On the semi-simplicity of the $U_{p}$-operator on modular forms, Math. Ann. 310 (1998), no. 1, 119-127.

[5] J. Cremona, Algorithms for modular elliptic curves, Cambridge University Press, Cambridge, second edition, 1997.

[6] M. Deuring, Die Zetafunktion einer algebraischen Kurve vom Geschlechte Eins, Nachr. Akad. Wiss. Gttingen. Math.-Phys. Kl. Math.-Phys.-Chem. Abt. (1953), 85-94.

[7] N. Elkies, The existence of infinitely many supersingular primes for every elliptic curve over $\mathbb{Q}$, Invent. Math. 89 (1987), no. 3, 561-567.

[8] F.Q. Gouvêa, Where the slopes are, J. Ramanujan Math. Soc. 16 (2001), no. 1, 75-99.

[9] K. Hatada, Eigenvalues of Hecke operators on $\mathrm{SL}(2, \mathbb{Z})$, Math. Ann. 239 (1979), no. $1,75-96$.

[10] E. Hecke, Über eine neue Art von Zetafunktionen und ihre Beziehungen zur Verteilungen der Primzahlen, Math. Z. 1 (1918), 357-376; 4 (1920), 11-21.

[11] H. Hida, On abelian varieties with complex multiplication as factors of the Jacobians of Shimura curves, Am. J. Math. 103 (1981) 727-776.

[12] H. Jacquet and R. Langlands, Automorphic forms on GL(2), Lecture Notes in Mathematics, Vol. 114, Springer-Verlag, Berlin, 1970.

[13] H. Kim and P. Sarnak, Refined estimates towards the Ramanujan and Selberg Conjectures, J. Amer. Math. Soc. 16 (2003), 139-183, Appendix to H. Kim, Functoriality for the exterior square of $\mathrm{GL}(4)$ and symmetric fourth of $\mathrm{GL}(2)$.

[14] H. Kim, F. Shahidi, Cuspidality of symmetric powers with applications, Duke Math. J. 112 (2002), no. 1, 177-197. 
[15] R. Livné, Communication networks and Hilbert modular forms, in Applications of algebraic geometry to coding theory, physics and computation, NATO Sci. Ser. II Math. Phys. Chem. 36, Kluwer Acad. Publ., Dordrecht, (2001), 255-270.

[16] The PARI Group, PARI/GP version 2.11.1, Univ. Bordeaux, 2018, http://pari. math.u-bordeaux.fr/.

[17] D. Ramakrishnan, On the coefficients of cusp forms, Math. Res. Lett., 4 (1997), 295-307.

[18] J. Rogawski, Functoriality and the Artin conjecture, in Representation theory and automorphic forms (Edinburgh, 1996), 331-353, Proc. Sympos. Pure Math., 61, Amer. Math. Soc., Providence, RI, 1997.

[19] J. Tate, Conjectures on algebraic cycles in $\ell$-adic cohomology, in Motives (Seattle, WA, 1991), 71-83, Proc. Sympos. Pure Math., 55, Part 1, Amer. Math. Soc., Providence, RI, 1994. 